\title{
Intermediate phases in mixed nematic/Heisenberg spin models
}

\author{
M Campbell and L Chayes \\ Department of Mathematics, University of California, Los Angeles, CA 90095-1555, USA \\ E-mail: mcampbe@math.ucla.edu and lchayes@math.ucla.edu
}

Received 16 February 1999, in final form 19 October 1999

\begin{abstract}
We prove that in three or higher dimensions, an isotropic, ferromagnetic, nearestneighbour perturbation of an $\mathrm{O}(N)$ nematic liquid crystal model has an intermediate phase. In this intermediate phase, the liquid crystal order parameter is non-zero but the spontaneous magnetization is zero while at low temperatures, these systems exhibit magnetization.
\end{abstract}

This paper concerns the (lattice) nematic liquid crystal model. Here, at each site $i$ of a regular lattice, resides an $N$-dimensional spin variable, $\vec{s}_{i}$. (We will take $\left|\vec{s}_{i}\right|=1$; for physical considerations, one focuses on $N=2$ or 3 .) This variable, in some approximation, is supposed to represent a rod-like molecule (or density thereof) that is symmetric about its centre. Thus the spin-spin interaction usually boils down to the square of the standard Heisenberg exchange interaction. If the coefficient is negative, the model is attractive; this is the case we consider. Such models can have a low-temperature phase, called the nematic phase. The nematic states are translation invariant and are characterized by the non-vanishing of the nematic order parameter, i.e. the matrix

$$
\mathbb{Q}_{i}^{\alpha, \gamma}=s_{i}^{\alpha} s_{j}^{\gamma}-\frac{1}{N} \delta^{\alpha \gamma}
$$

does not average to zero. Needless to say in these models the spins themselves have zero average at all temperatures.

Standard mean-field theory (see, e.g., [dGP]) indicates a first-order transition for $N \geqslant 3$ (but not for $N=2$ ). Presumably this is the case in dimension three and higher as indicated by simulations and Bethe lattice calculations [BZCP, KJ] (see also [dGP, CL] and references therein). Such models have also been investigated mathematically in [AZ,Z] (and more recently $[\mathrm{TI}]$; cf the remark below). For the standard nearest-neighbour model in $d \geqslant 3$, the existence of a nematic phase was established in the first reference. In [Z] the low-dimensional long-range versions of these systems were studied with similar success. (However, to the authors' knowledge there is, as of yet, no rigorous statement concerning the order of the transitions.)

Entirely different physics is exhibited in the 'antiferromagnetic' versions-where the coupling favours anti-alignment. We refer the interested reader to a sample of the extensive literature on this subject [BFMS, BFMS2, BFMS3, KS, R]. Unfortunately, except for the rather trivial case of $N=2$, these systems cannot be treated by the present methods. (The technical obstructions will be clarified below.)

In this paper, we wish to consider a situation where the liquid crystal molecules have some asymmetry along the axis of the rod, which will become physically relevant at sufficiently low 
temperatures. For example, the 'head' and 'tail' ends of the molecules may have different interactions that tend to align the heads of the molecules, as was discussed in [LG]. Thus the natural model to consider is the standard liquid crystal model (e.g. as written down in [AZ]) with the Hamiltonian perturbed by a Heisenberg term. Formally,

$$
-\mathcal{H}=J \sum_{\langle i, j\rangle} \operatorname{Tr}\left[\mathbb{Q}_{i} \mathbb{Q}_{j}\right]+\epsilon \sum_{\langle i, j\rangle}\left(\vec{s}_{i} \cdot \vec{s}_{j}\right) .
$$

This is precisely the sort of system that was analysed in [LG] by renormalization group methods for the case $N=2$ and $d=2$. There it was convincingly argued that the system exhibits an intermediate phase distinguished from the low- and high-temperature phases by the asymptotic behaviour of various correlation functions. Here, mostly for technical reasons, we will focus on $d \geqslant 3$. Then the techniques of reflection positivity and infrared bounds [FSS, FILS] tell us that both the liquid crystal and the magnetic order parameters have associated transition temperatures below which they are non-zero. The purpose of this paper is to show that these temperatures can be distinct, and consequently the existence of an intermediate phase for these systems as well. Of course in these cases, the intermediate phase is characterized by a positive nematic order parameter and zero magnetization. We use a generalized graphical representation of the type discussed in $[\mathrm{CM}]$ to distinguish these temperatures.

After the submission of this work, it was pointed out to us that nearly an identical problem had been studied in [TI] where many of the same results had been derived. However, there are various features that distinguish the present work from that of [TI]. The methods of proof of the principal result (section 5 in [TI], theorem 3 in the present work) are quite different. In [TI] it was assumed that the term causing the nematic order was of a very particular form-which is satisfied by the interaction in equation (2) - in order that it may be reduced to a ferromagnetic Ising system. In contrast, our argument only requires that: (a) the total Hamiltonian produces nematic order, and (b) the 'non-magnetic' portion is invariant under the reversal of any spin. In particular, suppose that the nematic interactions contain a (small) higher-order multi-pole disturbance. For example, the interaction in equation (2) is replaced by

$$
-\mathcal{H}=J \sum_{\langle i, j\rangle} \operatorname{Tr}\left[\left(\mathbf{Q}_{i}-\eta \mathbf{D}_{i}\right)\left(\mathbf{Q}_{j}-\eta \mathbf{D}_{i}\right)\right]+\epsilon \sum_{\langle i, j\rangle}\left(\vec{s}_{i} \cdot \vec{s}_{j}\right)
$$

where $\mathbf{Q}_{i}=\mathbb{Q}_{i} \otimes \mathbb{I}$ and $\mathbf{D}_{i}=\mathbb{S}_{i} \otimes \mathbb{S}_{i}-1 / N^{2} \mathbb{I} \otimes \mathbb{I}$, with $\mathbb{S}_{i}^{\alpha, \gamma} \equiv s_{i}^{\alpha} s_{i}^{\gamma}$. A 'nematic' phase for such a model is easily established using reflection positivity. Due to the mixed term, the purely ferromagnetic nature of the reduced Hamiltonian used in [TI] is ruined and the dipolar term cannot be controlled. Here, no such technical obstructions are encountered and the existence of the intermediate phase could be proved mutatis mutandis. Furthermore, as it turns out, the argument used here is extremely simple.

The set-up for this model in finite volume consists of a box $\Lambda \subset \mathbb{Z}^{d}, d \geqslant 3$, with bonds $\mathbb{B}$, and a 'liquid crystal rod' (i.e. an $\mathrm{O}(N)$ variable) centred at each site. The ends of the rod are antipodal points on the $(N-1)$-dimensional unit sphere $S^{N-1}$ and the (matrix) observable for this molecule is $\mathbb{Q}_{i}^{\alpha, \gamma}$ as defined in equation (1). Since we need reflection positivity techniques to ensure the existence of regions where the various order parameters are positive, we must implement some restrictions: first, the interaction itself must be quadratic-in some set of variables - and attractive. This is precisely what disallows the study of anti-nematic interactions by these methods $\dagger$. The second restriction is to nearest-neighbour interactions or to long-range interactions of a very particular type (as described in [FILS]). These sorts of long-range forces (in the present context) were treated in [Z] for the pure nematic problem

$\dagger$ The exceptional case is the $\mathrm{O}(2)$ anti-nematic. Here it is seen that a $90^{\circ}$ rotation of the (internal) axes on the odd or even sublattice results in an attractive nematic interaction. In the absence of further perturbations, this can be treated by the existing methods. 
and, with additional work, could be accommodated here. Nevertheless, in this work, we will confine attention to the simplest system that exhibits the intermediate phase.

Of course we will now have to use periodic boundary conditions. Thus, in the absence of spatially dependent external fields, the states we consider are automatically translation invariant, so the magnetization observable is equal to $\vec{s}_{0}$ and the nematic observable equal to $\mathbb{Q}_{0}$. The full Hamiltonian, including the Heisenberg perturbation and external fields, is given by

$\mathcal{H}_{\Lambda}(\vec{h}, \mathbb{K})=-\sum_{\langle i, j\rangle \in \mathbb{B}}\left(J \operatorname{Tr}\left[\mathbb{Q}_{i} \mathbb{Q}_{j}\right]+\epsilon\left(\vec{s}_{i} \cdot \vec{s}_{j}\right)\right)-\sum_{i \in \Lambda}\left(\operatorname{Tr}\left[\mathbb{K}_{i} \mathbb{Q}_{i}\right]+\vec{h}_{i} \cdot \vec{s}_{i}\right)$

with $\vec{s}_{i} \in S^{N-1}, \vec{h} \in \mathbb{R}^{N}$, and $\epsilon>0$. The objects $\mathbb{K}_{i}$ are traceless $N \times N$ self-adjoint matrices:

$$
\mathbb{K}_{i}^{\alpha \gamma}=k_{i}^{\alpha} k_{i}^{\gamma}-\frac{1}{N}\left|\vec{k}_{i}\right| \delta^{\alpha \gamma}
$$

where $\vec{k}_{i}$ is a vector like $\vec{h}_{i}$, with components $k_{i}^{\alpha}, \alpha=1, \ldots, N$. The external fields, $\mathbb{K} \equiv \mathbb{K}_{i}$ and $\vec{h} \equiv \vec{h}_{i}$ are for auxiliary purposes only and will not enter into the final results. The partition function is given by

$$
\mathcal{Z}_{\Lambda}(\vec{h}, \mathbb{K})=\int \mathrm{d}^{|\Lambda|} s \mathrm{e}^{-\beta \mathcal{H}_{\Lambda}(\vec{h}, \mathbb{K})}
$$

where d $s$ denotes the Haar measure on $S^{N-1}$. We let $\langle\cdot\rangle_{\Lambda ; \vec{h}, \mathbb{K}}$ denote the Gibbs measure corresponding to $\mathcal{Z}_{\Lambda}(\vec{h}, \mathbb{K})$. The order parameters that we will consider are the infinite volume quantities $\left\langle\vec{s}_{0}\right\rangle_{0^{+}, 0}$ and $\left\langle\mathbb{Q}_{0}\right\rangle_{0,0^{+}}$. In both cases, the zero-field limit is taken with uniform external fields pointing along a particular axis.

Using the techniques pioneered in [FSS, FILS], we have first-order phase transitions with respect to $\vec{h}$ and $\mathbb{K}$. Furthermore, we have lower bounds on the transition temperatures for each order parameter. We will denote the lower bounds on the transition temperatures for $\left\langle\vec{s}_{0}\right\rangle_{0^{+}, 0}$ and $\left\langle\mathbb{Q}_{0}\right\rangle_{0,0^{+}}$by $T_{*}^{\text {spin }}$ and $T_{*}^{l c}$, respectively. The actual transition temperatures (below which $\left\langle\vec{s}_{0}\right\rangle_{0^{+}, 0}$ and $\left\langle\mathbb{Q}_{0}\right\rangle_{0,0^{+}}$do not vanish and above which they are identically zero) will be denoted by $T_{c}^{\text {spin }}$ and $T_{c}^{l c}$, respectively. We summarize the current status in the following.

Proposition 1. In dimension $d \geqslant 3$, there is a first-order phase transition in each variable $\vec{h}$ and $\mathbb{K}$ with $\left\langle\vec{s}_{0}\right\rangle_{0^{+}, 0}$ and $\left\langle\mathbb{Q}_{0}\right\rangle_{0,0^{+}}$serving as the respective order parameters for these phase transitions. These quantities are non-zero when $T<T_{*}^{\text {spin }}$ and/or $T<T_{*}^{l c}$; here the lower bounds on the transition temperatures are related to the interaction constants by $T_{*}^{\text {spin }} \equiv 4 \epsilon /[N D]$ and $T_{*}^{l c} \equiv 8 J(N-1) /\left[N^{2}(N+1) D\right]$, where $D$ is the constant in [FILS, FSS]:

$$
D \equiv(2 \pi)^{-d} \int_{\substack{\left|p_{m}\right| \leqslant \pi \\ m=1, \ldots, d}}\left(\sum_{m=1}^{d} 1-\cos p_{m}\right)^{-1} \mathrm{~d}^{d} p .
$$

Furthermore, the temperatures $T_{c}^{\text {spin }}$ and $T_{c}^{l c}$ are finite, i.e. there is a genuine high-temperature phase.

Proof. The temperatures $T_{*}^{s p i n}$ and $T_{*}^{l c}$ are obtained by the methods in section 4 of [FILS]; we will outline the derivation in [AZ] for $T_{*}^{l c}$.

We use matrices that have a single non-zero entry on or above the diagonal at $(\alpha, \gamma)$ to obtain all of the mixed-term bounds for Gaussian domination: $\mathcal{Z}_{\Lambda}\left(0, \mathbb{H}^{\alpha \gamma}\right) \leqslant \mathcal{Z}_{\Lambda}(0,0)$. We have $N(N+1) / 2$ bounds (since there are that many elements on or above the diagonal of an 
$N \times N$ matrix). By summing these bounds and following the derivation in [FSS, FILS], we see that if

$$
\left\langle\operatorname{Tr}\left(\mathbb{Q}_{0}^{2}\right)\right\rangle-\frac{1}{4 \beta} \frac{N(N+1)}{2} J D \geqslant 0
$$

there will be multiple phases, and thus for some ergodic state $\mu, \mu\left(\mathbb{Q}_{0}\right) \neq 0$. This will happen if $T<[N(N+1) D]^{-1} 8 J\left\langle\operatorname{Tr}\left(\mathbb{Q}_{0}^{2}\right)\right\rangle=[N(N+1) D]^{-1} 8 J(N-1) / N$. The classic derivation (in [FSS]) results in the value for $T_{*}^{\text {spin }}$. Standard high-temperature techniques ([S, V.1.3], Dobrushin's uniqueness theorem or the method in [AC]) show that the temperatures $T_{c}^{\text {spin }}$ and $T_{c}^{l c}$ are finite.

We will now define a graphical representation that is useful for systems of the type described in equations (2). As a direct consequence we will obtain an upper bound on the temperature $T_{c}^{\text {spin }}$ (which, we recall is the temperature below which there is magnetic ordering). The goal of this paper is achieved if our upper bound lies below $T_{*}^{l c}$; this, we show, is indeed the case if $\epsilon / J$ is small.

Let us start this with some preliminary notation. As before, let $\Lambda \subset \mathbb{Z}^{d}$ be a finite box with bonds $\mathbb{B}$. Let $\mathcal{H}_{\Lambda}^{(0)}$ denote any interaction on configurations of spins $\left\{\vec{s}_{i} \mid i \in \Lambda\right\}$ that is invariant under the reversal of any spin:

$$
\mathcal{H}_{\Lambda}^{(0)}\left(\vec{s}_{1}, \ldots, \vec{s}_{j}, \ldots, \vec{s}_{|\Lambda|}\right)=\mathcal{H}_{\Lambda}^{(0)}\left(\vec{s}_{1}, \ldots,-\vec{s}_{j}, \ldots, \vec{s}_{|\Lambda|}\right) .
$$

We define

$$
\mathcal{H}_{\Lambda}^{\text {spin }}=-\sum_{\langle i, j\rangle \in \mathbb{B}} \epsilon\left(\vec{s}_{i} \cdot \vec{s}_{j}+1\right)
$$

and $\mathcal{H}_{\Lambda}=\mathcal{H}_{\Lambda}^{(0)}+\mathcal{H}_{\Lambda}^{\text {spin }}$. Here we will allow arbitrary boundary conditions on $\partial \Lambda$, but these will not enter into the notation. Observe that in the magnetic term, we have added a constant (which of course, has no physical consequences).

To find our graphical representation, we write

$$
\mathcal{Z}_{\Lambda}=\operatorname{Tr}\left[\mathrm{e}^{-\beta \mathcal{H}_{\Lambda}}\right]=\operatorname{Tr}\left[\mathrm{e}^{-\beta \mathcal{H}_{\Lambda}^{(0)}} \prod_{\langle i, j\rangle \in \mathbb{B}} \mathrm{e}^{\beta \epsilon\left(\vec{s}_{i} \cdot \vec{s}_{j}+1\right)}\right]
$$

where the operation of ' $\mathrm{Tr}$ ' includes any special considerations that take place at the boundary. We expand the product in equation (6) associating each term in the expansion with a bond configuration $\omega \in\{0,1\}^{\mathbb{B}}$. In particular, for each $\langle i, j\rangle$ we must 'choose' the term $\left(\mathrm{e}^{\beta \epsilon\left(\vec{s}_{i} \cdot \vec{s}_{j}+1\right)}-1\right)$ or +1 which corresponds to $\omega_{\langle i, j\rangle}=1$ (occupied) or $\omega_{\langle i, j\rangle}=0$ (vacant), respectively. It is noted that the contribution from each term in the sum is positive. Thus the weights

$$
V_{\Lambda}(\omega)=\int \mathrm{d}^{|\Lambda|} s \mathrm{e}^{-\beta \mathcal{H}_{\Lambda}^{(o)}} \prod_{\langle i, j\rangle \in \omega}\left(\mathrm{e}^{\beta \epsilon\left(\vec{s}_{i} \cdot \vec{s}_{j}+1\right)}-1\right)
$$

when divided by the partition function may be interpreted as the probability of observing the configuration $\omega$. We denote the associated probability measure by $v_{\Lambda}(-)$. We write $i \leftrightarrow \partial \Lambda$ if there is a path of occupied bonds in $\omega$ connecting the site $i$ to $\partial \Lambda$, and $i \nLeftarrow \partial \Lambda$ if there is no such path connecting $i$ to $\partial \Lambda$. Although the weights appear rather unwieldy it is straightforward to obtain bounds on certain probabilities. For example, we have:

Lemma 2. Let $\left\{e_{1}, e_{2}, \ldots, e_{n}\right\}$ denote a given set of edges. Then in any boundary condition, the probability that all of these edges are occupied, $v_{\Lambda}\left(e_{1}=1, e_{2}=1, \ldots, e_{n}=1\right)$, is bounded above by $\left(\mathrm{e}^{2 \beta \epsilon}-1\right)^{n}$. 
Proof. This is an elementary observation,

$$
\begin{aligned}
v_{\Lambda}\left(e_{1}=1, e_{2}=1, \ldots, e_{n}=1\right) & =\sum_{\substack{\omega: \omega\left(e_{m}\right)=1 \\
m=1, \ldots, n}} \mathcal{Z}_{\Lambda}^{-1} \int \mathrm{d}^{|\Lambda|} s \mathrm{e}^{-\beta \mathcal{H}_{\Lambda}^{(0)}} \prod_{\langle i, j\rangle \in \mathbb{B}}\left(\mathrm{e}^{\beta \epsilon\left(\vec{s}_{i} \cdot \vec{s}_{j}+1\right)}-1\right)^{\omega\langle i, j\rangle} \\
& \leqslant\left(\mathrm{e}^{2 \beta \epsilon}-1\right)^{n} v_{\Lambda}\left(e_{1}=0, e_{2}=0, \ldots, e_{n}=0\right) \\
& \leqslant\left(\mathrm{e}^{2 \beta \epsilon}-1\right)^{n}
\end{aligned}
$$

It is noted that the inequality in equation (8) is independent of boundary conditions.

This leads immediately to our central result:

Theorem 3. Consider the interaction $\mathcal{H}_{\Lambda}=\mathcal{H}_{\Lambda}^{(0)}+\mathcal{H}_{\Lambda}^{\text {spin }}$ as described above and let $T_{0}^{\text {spin }}$ be defined by $\exp \left\{2|\epsilon| / T_{0}^{\text {spin }}\right\}-1=\lambda^{-1}$ where $\lambda$ (which satisfies $\lambda<2 d-1$ on $\mathbb{Z}^{d}$ ) is the connectivity of the lattice. Then for $T>T_{0}^{\text {spin }}$ there is no magnetic ordering in any limiting state of this interaction.

Proof. Let $\Lambda$ be a finite box as above and let $\langle\cdot\rangle_{\Lambda}^{*}$ be the finite-volume Gibbs measure using the Hamiltonian $\mathcal{H}_{\Lambda}$ with boundary conditions $*$ and the temperature dependence suppressed. Then, by well known arguments (cf [S, ch III.3]) the spontaneous magnetization is bounded above by $\sup _{*}\left\langle s_{0}^{1}\right\rangle_{\Lambda}^{*}$. Thus it is sufficient to show that for large $\Lambda$, this quantity is small in any boundary condition.

Let us write, according to the preliminary steps of the graphical representation, the identity

$$
\left\langle s_{0}^{1}\right\rangle_{\Lambda, \beta}^{*}=\sum_{\omega \in\{0,1\}^{\mathbb{B}}} \mathcal{Z}_{\Lambda}^{-1} \int \mathrm{d}^{|\Lambda|} s \mathrm{e}^{-\beta \mathcal{H}_{\Lambda}^{(0)}} s_{0}^{1} \prod_{\langle i, j\rangle \in \omega}\left(\mathrm{e}^{\beta \epsilon\left(\vec{s}_{i} \cdot \vec{s}_{j}+1\right)}-1\right) .
$$

We claim that for any configuration $\omega$ such that $0 \nLeftarrow \partial \Lambda$, the corresponding term in equation (9) vanishes.

To see this let $C_{0}(\omega)$ denote the connected cluster of the origin in the configuration $\omega$. If $C_{0}(\omega)$ does not touch the boundary, then the integrals of all of the $\vec{s}_{k}, k \in C_{0}(\omega)$ are performed with respect to the full Haar measure. Now consider the change of variables $\vec{s}_{k} \mapsto-\vec{s}_{k}, k \in C_{0}(\omega)$. Obviously if $i$ and $j$ are both in $C_{0}(\omega)$, then the term $\vec{s}_{i} \cdot \vec{s}_{j}$ is unaffected. Further, since $\mathcal{H}_{\Lambda}^{(0)}$ is invariant under all spin reversals (by assumption) there is also no change (independent of whether or not $i \in C_{0}(\omega)$ ). On the other hand, this transformation will negate the $s_{0}^{1}$ that sits in front and thus the whole term cancels out.

Evidently, we are left with the more restrictive sum where the origin connects to the boundary:

$$
\left\langle s_{0}^{1}\right\rangle_{\Lambda, \beta}^{*}=\mathcal{Z}_{\Lambda}^{-1} \sum_{\omega: 0 \leftrightarrow \partial \Lambda} \int \mathrm{d}^{|\Lambda|} s \mathrm{e}^{-\beta \mathcal{H}_{\Lambda}^{(0)}} s_{0}^{1} \prod_{\langle i, j\rangle \in \mathbb{B}}\left(\mathrm{e}^{\beta \epsilon\left(\vec{s}_{i} \cdot \vec{s}_{j}+1\right)}-1\right) .
$$

Since $\left|s_{0}^{1}\right| \leqslant 1$, we see that

$$
\left|\left\langle s_{0}^{1}\right\rangle_{\Lambda, \beta}^{*}\right| \leqslant v_{\Lambda}(0 \leftrightarrow \partial \Lambda)
$$

where on the right-hand side we again suppress in our notation any dependence on boundary conditions.

At this point all we need is a Peierls' argument. Any configuration $\omega$ with $0 \leftrightarrow \partial \Lambda$ must have a connected path of occupied bonds (a self-avoiding walk) between the origin and the boundary. Let $R$ denote the distance between the origin and the boundary. If $\left\{e_{1}, e_{2}, \ldots, e_{S}\right\}$ 
(with $S \geqslant R$ ) is one such walk, let $\mathcal{W}\left(e_{1}, e_{2}, \ldots, e_{S}\right)$ denote the event that all these edges are occupied. Using the result of lemma 2 we have

$$
\begin{aligned}
v_{\Lambda}^{*}(0 \leftrightarrow \partial \Lambda) \leqslant & \sum_{\substack{\text { all walks, } S \geqslant R \\
\mathcal{W}\left(e_{1}, e_{2}, \ldots, e_{S}\right)}} v_{\Lambda}^{*}\left(\mathcal{W}\left(e_{1}, e_{2}, \ldots, e_{S}\right)\right) \\
\leqslant & \sum_{\substack{\text { all walks, } S \geqslant R \\
\mathcal{W}\left(e_{1}, e_{2}, \ldots, e_{S}\right)}}\left(\mathrm{e}^{2 \beta \epsilon}-1\right)^{S} \\
\leqslant & C\left(\mathrm{e}^{2 \beta \epsilon}-1\right)^{R} \lambda^{R}
\end{aligned}
$$

for some constant $C$ independent of $R$ that is finite if $\left(\mathrm{e}^{2 \beta \epsilon}-1\right) \lambda<1$. Since these arguments are uniform in $|\Lambda|$ and independent of boundary conditions it follows immediately that when $\left(\mathrm{e}^{2 \beta \epsilon}-1\right) \lambda<1$, the spontaneous magnetization vanishes.

Remark. Improved values for $T_{0}^{\text {spin }}$ can be obtained. Indeed, on the basis of just a little more effort, it can be established that the measures $v_{\Lambda}(-)$ are dominated by independent (Bernoulli) measures with parameter $p=1-\mathrm{e}^{-2 \beta \epsilon}$. This may be derived by going to a full blown EdwardsSokal measure [ES] along with an associated cluster algorithm. Then, at each bond step, regardless of the spin configuration, one notices that the maximum bond probability can never exceed $1-\mathrm{e}^{-2 \beta \epsilon}$. The upshot is that the improved $T_{0}^{\text {spin }}$ satisfies $1-\exp \left\{-2 \epsilon / T_{0}^{\text {spin }}\right\}=p_{c}(d)$ where $p_{c}(d)$ is the bond percolation threshold for the $d$-dimensional lattice.

Corollary. Consider the Hamiltonian described in equation (2) in $d \geqslant 3$. Then if $\epsilon / J$ is sufficiently small (depending on $N$ and $d$ ) there is an intermediate phase where the magnetization is zero and the nematic order parameter is positive.

Proof. To demonstrate the above, we need only apply theorem 3 to this case and find a 'window' between $T_{*}^{l c}$ and $T_{0}^{\text {spin }}$. Since, (for fixed $N$ and $d$ ) the former scales with $J$ and the latter with $\epsilon$ (for $\epsilon \ll 1)$, the desired result follows immediately.

\section{References}

[AC] Alexander K and Chayes L 1997 Non-perturbative criteria for Gibbsian uniqueness Commun. Math. Phys. $189447-64$

[AZ] Angelescu N and Zagrebnov V A 1982 A lattice model of liquid crystals with matrix order parameter $J$. Phys. A: Math. Gen. 15 L639-43

[BFMS] Ballesteros H G, Fernández L A, Martín-Mayor V V and Muñoz Sudupe A 1996 New universality class in three dimensions?: the antiferromagnetic $R P^{2}$ model Phys. Lett. B 378 207-12

[BFMS2] Ballesteros H G, Fernández L A, Martín-Mayor V V and Muñoz Sudupe A 1997 Critical properties of the antiferromagnetic $R P^{2}$ model in three dimensions Nucl. Phys. B $483707-36$

[BFMS3] Ballesteros H G, Fernández L A, Martín-Mayor V V and Muñoz Sudupe A 1997 Critical exponents and unusual properties of the broken phase in the $3 \mathrm{~d}-R P^{2}$ antiferromagnetic model Nucl. Phys. B (Proc. Suppl.) $53686-9$

[BZCP] Biscarini F, Zannoni C, Chiccoli C and Pasini P 1991 Head-tail asymmetry and ferroelectricity in uniaxial liquid crystals Mol. Phys. 73 439-61

[CL] Chaikin P M and Lubensky T C 1995 Principles of Condensed Matter Physics (Cambridge: Cambridge University Press)

[CM] Chayes L and Machta J 1997 Graphical representations and cluster algorithms I. Discrete spin systems Physica A 239 542-601

[dGP] deGennes P D and Prost J 1993 The Physics of Liquid Crystals 2nd edn (Oxford: Clarenden)

[ES] Edwards R G and Sokal A D 1988 Generalization of the Fortuin-Kasteleyn-Swendsen-Wang representation and Monte Carlo algorithm Phys. Rev. D 38 2009-12 
[FILS] Frölich J, Israel R, Lieb E H and Simon B 1978 Phase transitions and reflection positivity. I. General theory and long range lattice models Commun. Math. Phys. 62 1-34

[FSS] Fröhlich J, Simon B and Spencer T 1978 Infrared bounds, phase transitions and continuous symmetry breaking Commun. Math. Phys. 62134

[KJ] Krieger T J and James H M 1982 Successive orientational transitions in crystals J. Phys. A: Math. Gen. 15 L639-43

[KS] Kohring G and Shrock R E 1988 Effects of ground state disorder without frustration Nucl. Phys. B 295 $36-50$

[LG] Lee D H and Grinstein G 1985 Strings in two-dimensional classical XY models Phys. Rev. Lett. 55 541-4

[R] Romano S 1994 Computer simulation study of a three-dimensional lattice spin model with anti-nematic interactions Int. J. Mod. Phys. B 8 3389-410

[S] Simon B 1993 The Statistical Mechanics of Lattice Gases vol 1 (Princeton, NJ: Princeton University Press)

[TI] Tanaka A and Idogaki T 1998 Long range orders on the classical bilinear-biquadratic exchange Hamiltonian J. Phys. Soc. Japan 67 604-11

[Z] Zagrebnov V A 1996 Long-range order in a lattice gas model of nematic liquid crystals Physica A 232 737-46 